\title{
Ceramide Nanoliposome
}

National Cancer Institute

\section{Source}

National Cancer Institute. Ceramide Nanoliposome. NCI Thesaurus. Code C131434.

A lipid-based nanoparticle formulation composed of the apoptosis-inducing sphingolipid ceramide encapsulated within nanoliposomes, with potential apoptotic and antineoplastic activities. Upon administration, ceramide nanoliposomes accumulate in the tumor environment, due to the unique properties of the tumor vasculature, and easily enter tumor cells. This delivers ceramide inside the tumor cells, where ceramide induces apoptosis. Although the process is not completely understood, ceramide-dependent apoptosis most likely results from the downregulation of nutrient transporter proteins, which prevents cellular access to extracellular nutrients and causes tumor cell starvation. This selectively destroys tumor cells. Ceramide alone is insoluble and has a very short half-life; therefore, the nanoliposome formulation increases its solubility and half-life. Ceramide plays a key role in the regulation of autophagy, apoptosis, survival and proliferation. Serving as a tumor suppressor lipid, the expression of ceramide is inversely correlated with tumor cell growth, survival and metastasis. 